\title{
Cholinergic Augmentation Modulates Visual Task Performance in Sleep-Deprived Young Adults
}

\author{
Lisa Y. M. Chuah and Michael W. L. Chee \\ Cognitive Neuroscience Laboratory, Duke-National University of Singapore Graduate Medical School, Singapore 169611
}

Using $24 \mathrm{~h}$ of total sleep deprivation to perturb normal cognitive function, we conducted a double-blind, placebo-controlled crossover study to evaluate the effect of the acetylcholinesterase inhibitor, donepezil, on behavioral performance and task-related brain activation in 28 healthy, young, adult volunteers. The behavioral tasks involved the parametric manipulation of visual short-term memory load and perceptual load in separate experiments indirectly evaluating attention. Sleep deprivation significantly reduced posterior cortical activation (intraparietal sulcus and extrastriate cortex) at all levels of visual memory as well as perceptual load. Donepezil modulated an individual's performance in both tasks in accordance to whether accuracy declined after sleep deprivation without treatment. Critically, there were significant correlations between donepezil-induced increases in neural activation in the posterior cortical areas and improvement in accuracy. Reduced visual short-term memory after sleep deprivation may thus originate from a decline in visual attention and/or visual processing. Cholinergic augmentation can alleviate these deficits in individuals vulnerable to the effects of sleep deprivation, but it may have neutral or negative effects on those resistant to sleep deprivation.

Key words: visual cortex; parietal cortex, fMRI; visual short-term memory; attention; sleep deprivation; cholinergic system

\section{Introduction}

Short-term total sleep deprivation (SD) can result in cognitive impairments (Durmer and Dinges, 2005) that contribute to industrial catastrophes, transportation accidents, and medical errors (Mitler et al., 1988; Dinges, 1995; Barger et al., 2006; Philip and Akerstedt, 2006). This has motivated the use of pharmacological countermeasures, the most widely used being caffeine (Bonnet et al., 2005). Functional imaging studies of sleepdeprived persons could provide information concerning the neural substrates associated with SD-induced cognitive decline and, in addition, provide functional assays for assessing drug effects. However, despite the growing number of functional imaging studies involving SD (Chee and Chuah, 2008), neuropharmacological studies in this context are rare (Thomas and Kwong, 2006).

Impairment of attention in sleep-deprived persons (Lim and Dinges, 2008) is an important cognitive deficit that affects other "higher" cognitive operations, for example, visual short-term memory (VSTM) (Chee and Chuah, 2007). VSTM is used in service of many higher cognitive operations and has a capacity limit (Luck and Vogel, 1997; Cowan, 2001) that has neuroimag-

\footnotetext{
Received Aug. 25, 2008; revised Sept. 18, 2008; accepted Sept. 21, 2008.

This work was supported by the Neuroscience Centre of Excellence of Drug Discovery, GlaxoSmithKline (Contract Number 007091), and Defence Science and Technology Agency, Singapore (P0D0713897). Annette Chen, Delise Chong, William Rekshan III, and Enhui Yong performed scans and were involved in data preprocessing. Annette Chen helped prepare illustrations. Vincenzo Libri, Robert Lai, and Martin Pan from GlaxoSmithKline provided helpful comments and discussions.

This article is freely available online through the J Neurosci Open Choice option.

Correspondence should be addressed to Dr. Michael W. L. Chee, Cognitive Neuroscience Laboratory, DukeNational University of Singapore Graduate Medical School, 7 Hospital Drive, \#01-11, Block B, Singapore 169611. E-mail:mchee@pacific.net.sg.

DOI:10.1523/JNEUROSCI.4045-08.2008

Copyright $\odot 2008$ Society for Neuroscience 0270-6474/08/2811369-09\$15.00/0
}

ing correlates (Linden et al., 2003; Majerus et al., 2007; Mitchell and Cusack, 2007). SD-induced decline in VSTM capacity has been shown to originate from depressed visual attention or visual processing (Chee and Chuah, 2007). This was inferred from observing state-dependent, but load-independent, attenuation of parieto-occipital activation elicited by two visual tasks in which memory and perceptual load were independently and parametrically varied. Critically, the load-independent manner in which $\mathrm{SD}$ affected parieto-occipital activation across the tasks signified that something more fundamental than memory was depressed.

We reasoned that the decline in attention/visual processing might arise from reduced noradrenergic and cholinergic drive, given that these neurotransmitters underlie the maintenance of wakefulness (Everitt and Robbins, 1997; Steriade and McCarley, 2005) as well as visuospatial attention (Everitt and Robbins, 1997; Phillips et al., 2000). Increasing cholinergic transmission may improve visual working memory by increasing the selectivity of perceptual processing in the visual cortex (Furey et al., 2000b). Other experiments on visual memory have suggested that behavioral benefit may be associated with increases in extrastriate (Furey et al., 2000b; Lawrence et al., 2002) and superior parietal (Mentis et al., 2001) regions. Conversely, cholinergic blockade using scopolamine may impair orienting responses that involve parietal regions (Davidson and Marrocco, 2000). Of relevance to the present study, the concentration of cholinergic effects in the parieto-occipital region coincides with the locus of state effects in several functional imaging studies of short-term memory (Habeck et al., 2004; Chee and Chuah, 2007; Lim et al., 2007) in sleep-deprived persons.

Collectively, these findings led us to hypothesize that SDinduced deficits in visual processing/attention might benefit from cholinergic augmentation. We anticipated that the neural 
correlates of such performance modulation could be tracked by observing taskrelated parieto-occipital activation. To evaluate these hypotheses, we conducted a double-blind, placebo-controlled, crossover functional imaging study (see Fig. 1) to characterize the effect of donepezil on healthy young adults. In this withinsubject design, volunteers were scanned four times, twice after a normal night of sleep and twice after $24 \mathrm{~h}$ of total SD. In each scanning session, they performed two visual tasks that reliably reveal the effects of SD on attention across multiple levels of memory and perceptual load (see Fig. 2).

Mindful that cognitive deficits experienced after SD show interindividual variation (Leproult et al., 2003; Van Dongen et al., 2004), we studied a relatively large number of volunteers. Recent work with cholinergic agents in elderly volunteers (Beglinger et al., 2005; Bentley et al., 2008) has reinforced the notion that there exists a bell-shaped response curve to neurotransmitter augmentation (Honey and Bullmore, 2004). As such, we anticipated that the cognitive effects elicited by donepezil would correlate with how an individual performed while sleep deprived without cholinergic augmentation.

\section{Materials and Methods}

Participants

Forty-three healthy young adults were recruited through web-based advertisements and were screened by questionnaire and an interview. Prospective participants had to (1) be right-handed, (2) be between 18 and 35 years of age, (3) have habitual good sleeping habits (sleeping no less than $6.5 \mathrm{~h}$ each night for the past 1 month), (4) score no more than 22 on the Morningness-Eveningness Scale (Horne and Ostberg, 1976), (6) have no history of sleep disorder, (7) have no history of any psychiatric or neurologic disorders, (8) have normal color vision, (9) drink $<3$ cups of coffee a day, and (10) not smoke cigarettes. The sleeping habits of qualifying participants were monitored using wrist actigraphy, and only those who had good sleep habits (i.e., they usually slept no later than 1:00 A.M. and woke no later than 9:00 A.M.) were eligible for brain imaging. All participants were screened for color blindness and cleared blood and urine biochemistry tests before enrollment.

Twenty-eight participants (13 males; mean age, 22.14 years; SD, 1.01 years) successfully completed the study. Of the 15 who did not complete, 4 were screened out, 3 were unsuitable for magnetic resonance (MR) scanning (claustrophobia; large braces), 4 reported gastrointestinal side effects, and the remaining 4 were unable to maintain a regular sleepwake schedule. Participants were financially compensated for their time.

\section{Design and procedure}

The $2 \times 2$, double-blind, placebo-controlled, crossover study involved seven visits to the laboratory over a period of $\sim 2$ months (Fig. 1 ). Three of these visits were briefing sessions. The four remaining sessions were test/scanning sessions. Two of these test sessions were conducted after a night of normal sleep [rested wakefulness (RW)], and the remaining two sessions followed a night of SD. The order of the scanning sessions (rested wakefulness followed by sleep deprivation and vice versa) was counterbalanced across all subjects. A pharmacist who was not involved in data collection or analysis dispensed the tablets in a counterbalanced manner across all subjects. Compliance to the drug schedule was checked daily by telephone and further verified with tablet counts that were conducted before every scanning session.

During briefing session 1 (B1), participants were provided with detailed information about the aims and requirements of the study and gave informed consent before undergoing a physical examination and blood tests.

Briefing session 2 (B2) took place $\sim 3 \mathrm{~d}$ after B1 and involved subjects who passed the medical and laboratory test screens. Participants' sleeping patterns were verified by sleep diary and wrist actigraphy. During this session, participants practiced the tasks that were to be performed inscanner. At the end of this session, participants were given tablets (either $5 \mathrm{mg}$ donepezil or placebo) with instructions to take one a day at 9:00 A.M. and to record this. Participants took either $5 \mathrm{mg}$ of donepezil, or placebo, daily for an average of $10 \mathrm{~d}$ before their first scan session and continued taking the same tablets for another week until the end of the second scanning session. There was a 3 week washout period between each drug phase.

Briefing session 3 (B3), after washout, took place $\sim 3$ weeks after the second scanning session. Participants collected tablets and their compliance with the sleep schedule was verified. During the second drug phase, subjects took either donepezil or placebo daily for an average of $10 \mathrm{~d}$ before the third scan and continued taking the same tablets daily for a week until their fourth scan.

Rested wakefulness sessions. Scanning always took place at fixed times for both RW and SD sessions. For the RW sessions subjects arrived at the lab at 7:45 A.M. Compliance to the required sleep schedule was verified by checking the volunteer's sleep diary and wrist actigraph. All participants verified that they had not smoked, consumed any medications, stimulants, alcohol, or caffeine for at least $24 \mathrm{~h}$ before scanning. They also completed the Karolinska Sleepiness Scale (KSS) (Akerstedt and Gillberg, 1990) and performed a $10 \mathrm{~min}$ Psychomotor Vigilance Test (PVT) (Dinges et al., 1997; Doran et al., 2001).

Participants were scanned while performing the VSTM and visual perceptual control (VPC) tasks. The order of the two tasks was counterbalanced across subjects and scanning sessions. Before undergoing functional neuroimaging, participants were practiced on 12 trials of each task (two at each set size). During scanning, participants were prompted to respond through the intercom system when they failed to respond to two consecutive trials. A KSS rating was taken after the practice run and after each in-scanner run (four runs for each task).

Sleep deprivation sessions. Subjects reported to the laboratory at no later than 7:00 P.M. on the test night after staying awake the whole day. Sleep habits and compliance with tablet intake were checked as described for the RW session. Participants were monitored throughout the night and were only allowed to engage in nonstrenuous activities such as reading, working on a computer, and conversing. Every hour from 8:00 P.M. to 5:00 A.M., participants also completed the KSS and a 10 min PVT trial. Scanning took place at $\sim 5: 30$ A.M., close to or at the circadian nadir for most persons as described for the rested wakefulness session. Although it can be argued that our study does not purely reflect the effects of sleep deprivation but also includes circadian effects, most accidents occur at this time when persons are sleep-deprived (Horne and Reyner, 1995).

\section{Experimental tasks}

Visual short-term memory. Participants viewed and remembered arrays of up to eight differentiable colors. Array sizes of 1, 2, 3, 4, 6, and 8 were used. The stimuli were black-outlined, colored squares on a white background that occupied up to eight possible locations (in an invisible $3 \times 3$ grid, excluding the middle location) and subtended a maximum visual angle of $4 \times 4^{\circ}$. There was a pool of nine possible colors.

During each trial, an array was presented for $500 \mathrm{~ms}$ followed by a single memory probe $1000 \mathrm{~ms}$ later (Fig. 2A). Participants had $3000 \mathrm{~ms}$ to indicate whether the probe color was present/absent from the memory array by pressing a "yes" or "no" button. Each participant completed four runs of the task. Each run consisted of 48 trials, comprising eight trials of each set size. The trials were presented in an event-related design, with 


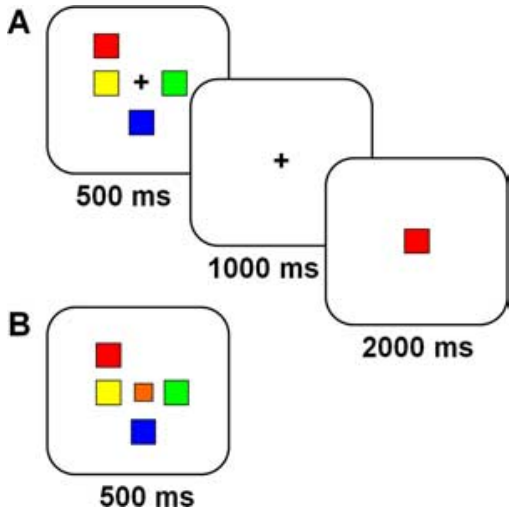

Figure 2. Schematic illustration of the VSTM $(\boldsymbol{A})$ and VPC $(\boldsymbol{B})$ tasks. For the VSTM task, subjects viewed and remembered arrays of up to eight colored squares. A single colored probe was presented $1 \mathrm{~s}$ later and subjects made a yes/no judgment as to whether the color was present in the most recently viewed array. There were four runs of the VSTM task and a total of 192 trials with 32 trials of each set size $(1,2,3,4,6$, and 8$)$. The intertrial interval was randomized to be $5,7.5$, or $10 \mathrm{~s}$ with equal probability of occurrence. In the VPC task, subjects were presented with identical visual arrays, but the task was to press "yes" if a square appeared in the center of the array and " $\mathrm{no}$ " otherwise.

random intertrial intervals of 5, 7.5, and $10 \mathrm{~s}$. Each run started with $10 \mathrm{~s}$ of fixation (data for the first $5 \mathrm{~s}$ was discarded to allow for steady-state magnetization) and ended with fixation lasting at least $17.5 \mathrm{~s}$.

Visual perceptual control. In this task, participants were presented with similar visual arrays of up to eight colors. Similar to the VSTM task, there were four runs of this task. Each run consisted of 48 trials, with 8 trials of each set size, presented in a random, event-related manner. For each trial, an array appeared for $500 \mathrm{~ms}$ and subjects responded immediately as to the presence or absence of a square at the center of the array (Fig. $2 B$ ). Subjects had $3000 \mathrm{~ms}$ to respond. The intertrial timing parameters were identical to the VSTM task.

Parametric variation of visual memory and perceptual load was originally designed to disambiguate changes in task-related activation linked to increased memory and perceptual load. Activation in the intraparietal sulcus (IPS) in the VSTM task has been shown to track storage, whereas extrastriate activation associated with both tasks reflects increased perceptual demands (Todd and Marois, 2004; Chee and Chuah, 2007). However, part of the IPS activation in VSTM-type tasks can also be attributed to focusing attention (Majerus et al., 2007; Mitchell and Cusack, 2007). These previous studies compared activation in the IPS across several VSTM tasks performed by the same individuals that tapped on common as well as distinct cognitive processes. This enabled investigators to tease apart the contribution of attention in IPS activation. Here, we used sleep deprivation to alter the context under which cognitive tasks were performed to reveal the contribution of attention.

\section{Imaging procedure and analysis}

Stimuli were projected onto a screen using a LCD projector (Epson EMP 7250; Epson) and viewed through a rearview mirror. Participants responded using a MR-compatible button box held in the right hand. A bite bar and foam padding were used to reduce head motion. Images were acquired on a $3 \mathrm{~T}$ Siemens Allegra system (Siemens). A gradient echo-planar imaging sequence was used with a repetition time of 2500 $\mathrm{ms}$, field of view of $192 \times 192 \mathrm{~mm}$, and a $64 \times 64 \mathrm{~mm}$ pixel matrix. Thirty-six oblique axial slices ( $3 \mathrm{~mm}$ thick with a $0.3 \mathrm{~mm}$ interslice gap) approximately parallel to the intercommisural plane were acquired.

Functional images were processed using Brain Voyager QX version 1.8.6 (Brain Innovation). Intrasession image alignment to correct for motion across runs was performed using, as the reference image, the first image of the functional run that was acquired immediately before the coplanar T1-weighted image. Interslice timing differences attributable to slice acquisition order were adjusted using linear interpolation. Gaussian filtering was applied in the spatial domain using a smoothing kernel of 8 $\mathrm{mm}$ full width at half-maximum for group-level activation maps. After linear trend removal, a high-pass filter of period $120 \mathrm{~s}$ was applied. The T1 images were used to register the functional data set to the volunteers' own high-resolution three-dimensional T1-weighted MPRAGE (magnetization-prepared rapid-acquisition gradient echo) image, and the resulting aligned dataset was transformed into Talairach space.

The functional data were analyzed for all four sessions using a general linear model with a total of 28 predictors. Cortical responses associated with correct responses were modeled using one predictor per set size for each of the four scan sessions. A separate predictor accounted for the incorrect trials in each session regardless of set size. All predictors were convolved with a canonical hemodynamic response function, and a mixed-effects analysis was used to identify significant voxels.

\section{Data analysis}

For both tasks, the effects of state, drug, and set size on performance accuracy were investigated using $2($ drug $) \times 2$ (state) $\times 6$ (set size) repeated-measures ANOVA. Accuracy was computed on the basis of all trials, including trials in which subjects failed to make a response within $3000 \mathrm{~ms}$ (nonresponses). Because there were no meaningful interactions between set size and either state or drug (supplemental material, available at www.jneurosci.org), we used averaged accuracy across all six set sizes for computation of Pearson's correlations that evaluated the association between donepezil-induced performance change and the performance decline wrought by sleep deprivation in the placebo condition.

Analysis of the imaging data focused on regions of interest (ROIs) identified by previous work as being modulated by set size, that is, reflecting the effects of memory and perceptual load as opposed to nonspecific aspects of task processing. The ROIs were identified using a 2 (drug) by 2 (state) by 6 (set size) repeated-measures ANOVA. Voxels surpassing the initial liberal threshold of $p<0.01$ underwent an iterative cluster thresholding procedure that considered the smoothness of the spatial map (Goebel et al., 2006) to compute a spatial map based on a corrected cluster threshold $(p<0.05)$. Only clusters that surpassed the voxelbased threshold of $p<0.05$ (corrected) were analyzed further.

As was the case for behavioral data, there was a general absence of significant interaction between set size and either state or drug (supplemental material, available at www.jneurosci.org). This afforded the use of an averaged activation metric to compute the correlation between changes in brain activation and behavioral accuracy elicited by donepezil in the context of sleep deprivation. All correlations were examined for possible bivariate outliers (Cook's distance, $>1$ ), and only correlations without such outliers were reported.

\section{Results}

\section{Behavioral findings}

Replicating previous findings (Chee and Chuah, 2007), we found that SD induced significant decline in accuracy associated with the VSTM task $\left(F_{(1,27)}=36.95 ; p<0.001\right)$. There was a similar performance decline associated with the VPC task $\left(F_{(1,27)}=\right.$ 63.65; $p<0.001$ ) (see supplemental material and supplemental Table 1, available at www.jneurosci.org as supplemental material). Supporting the hypothesis that the effects of SD on performance were mediated through lowered attention, there were significant correlations between several measures of performance decline in both experimental tasks and PVT lapses (reaction time $>500 \mathrm{~ms}$ ) (supplemental Table 4, available at www.jneurosci.org as supplemental material).

Donepezil had a beneficial effect in some individuals when they were sleep deprived but had no significant effect in the normal sleep condition (supplemental material, available at www. jneurosci.org). There was a significant correlation between an individual's performance decline after being sleep deprived without treatment and donepezil-related benefit (Fig. 3). This was true for both tasks (VSTM, $r=-0.65, p<0.001$; VPC, $r=$ $-0.60, p=0.001$; intertask correlation of donepezil-mediated benefit, $r=0.40, p=0.04$ ). Additional analyses with ANOVA (supplemental material, available at www.jneurosci.org) con- 
curred with the correlation analysis in showing that donepezil only modulated performance after SD. Moreover, although a benefit of drug was present for those most vulnerable to SD, those least vulnerable to SD experienced depressed performance (Fig. 4; supplemental material, available at www.jneurosci.org). In sum, across the two tasks and using different analyses, we found a bell-shaped response to donepezil in the context of SD across volunteers who differed in their sensitivity to cognitive decline when sleep deprived.

As expected, volunteers felt sleepier when they were sleep deprived $(p<0.001$ for the VSTM and VPC tasks) (supplemental Table 5, available at www. jneurosci.org as supplemental material). However, the absence of significant correlation between changes in self-reported sleepiness and performance improvement in either experimental task (largest correlation, $0.22 ; p=0.27$ ) suggests that donepezil-related performance gains were unlikely to arise from a reduction in sleepiness.

\section{Neuroimaging findings}

After a normal night of sleep, across drug conditions and as predicted, we observed capacity-limited IPS activation in the VSTM condition (Fig. 5; supplemental Fig. 1, available at www.jneurosci.org as supplemental material). The IPS response was significantly attenuated at all levels of memory load after sleep deprivation. This result is noteworthy because nonresponses were omitted from the analysis, excluding trials in which it could be argued that the volunteer was momentarily asleep.

Because the IPS participates in several cognitive processes depending on task demands and because of conflicting reports as to whether greater (Gray et al., 2003; Chee et al., 2004) or lesser (Rypma et al., 2006) activation constitutes a behaviorally advantageous pattern of neural activation, it was necessary to establish the functional relevance of IPS activation in the current experiments. After a normal night of sleep (RW), VSTM-related IPS activation correlated with accuracy in both placebo and drug conditions (RWP, $r=0.52, p=0.004$; RWD, $r=0.42, p=0.03$ ). Second, under placebo, $S D$-induced decline in performance accuracy correlated with reduced right IPS, bilateral middle occipital gyrus and left inferior frontal activation (Fig. 6).

To characterize the effects of donepezil on task-related activation and its relationship with performance, we examined the correlation between change in performance and brain activation in hypothesis-driven ROIs in the IPS and extrastriate cortex. Significant correlations were found between donepezil-induced improvement in performance in both experimental tasks and corresponding changes in brain activation within the IPS and extrastriate visual cortex (Figs. 6, 7). These regions overlapped
B. Visual Perceptual Control

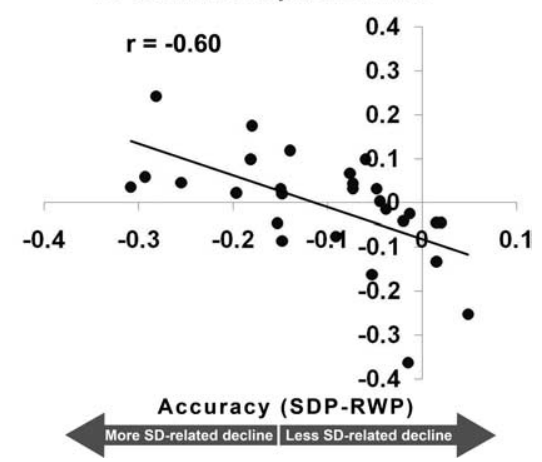

Figure 3. Correlation between the decline in performance accuracy after sleep deprivation relative to rested wakefulness in the placebo condition (SDP-RWP) and the extent of improvement with donepezil relative to placebo when sleep-deprived (SDD-SDP)

A. Visual Short-Term Memory

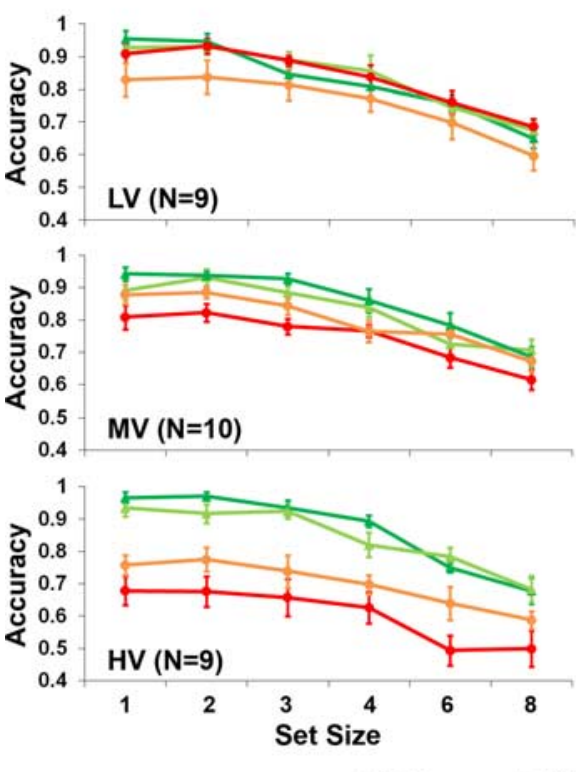

\section{B. Visual Perceptual Control}

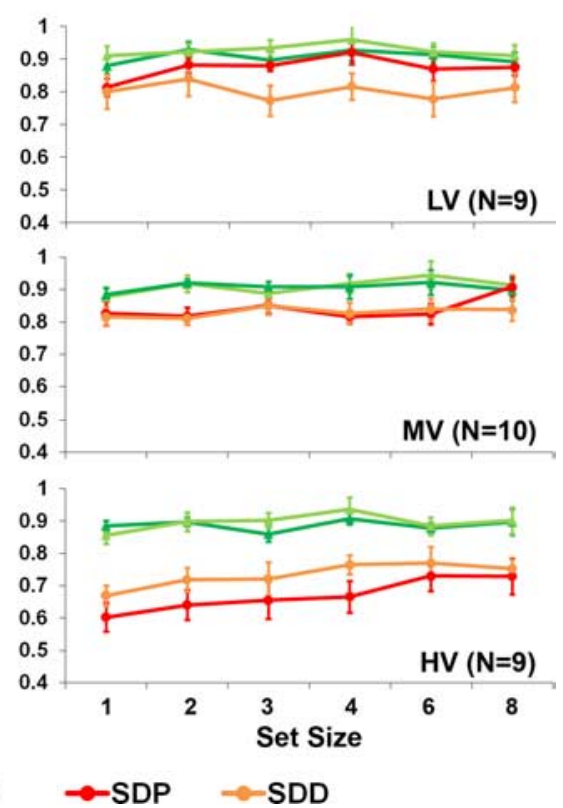

Figure 4. $\quad \boldsymbol{A}, \boldsymbol{B}$, Accuracy ( $\pm 1 \mathrm{SEM})$ associated with the visual short-term memory $(\boldsymbol{A})$ and visual perceptual control $(\boldsymbol{B})$ tasks during RW and SD as a function of set size, drug (P, placebo; D, donepezil) and group (LV, low vulnerability; MV, moderate vulnerability; HV, high vulnerability). These groups were derived based on the extent of SD-related change in number of nonresponses in the placebo condition across both tasks (see supplemental material, available at www.jneurosci.org).

with those whose activity tracked behavior decline after sleep deprivation in the placebo condition. In other words, the brain regions sensitive to the effects of sleep deprivation were also areas exhibiting a drug effect. Finally, there were also significant correlations pertaining to the association between donepezil-induced increments in activation when volunteers were sleep deprived and SD-induced declines in activation in the placebo condition (Figs. 6, 7). This suggests that neuroimaging results in the placebo condition can contribute to predicting the imaging response to donepezil under conditions of sleep deprivation.

In addition to these hypothesis-driven ROIs, donepezil modulated brain activation-behavior correlation in the left prefrontal region (see supplemental material, available at www.jneurosci. org). Donepezil increased prefrontal activation in sleep-deprived individuals in whom drug behavioral benefit, relative to placebo, was noted. This contrasts with previous findings using a different visual task in which cholinergic augmentation with physostig- 

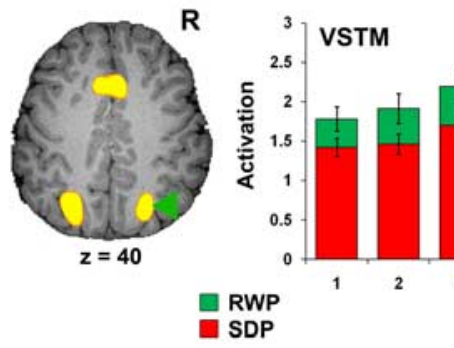

A. R Intraparietal Sulcus

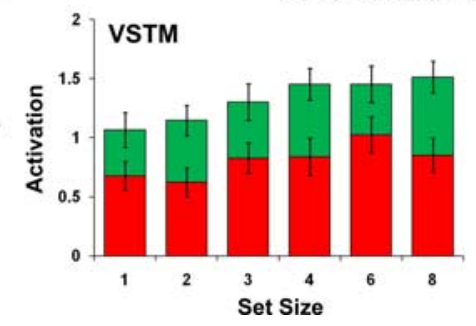

B. R Ventral Occipital Cortex
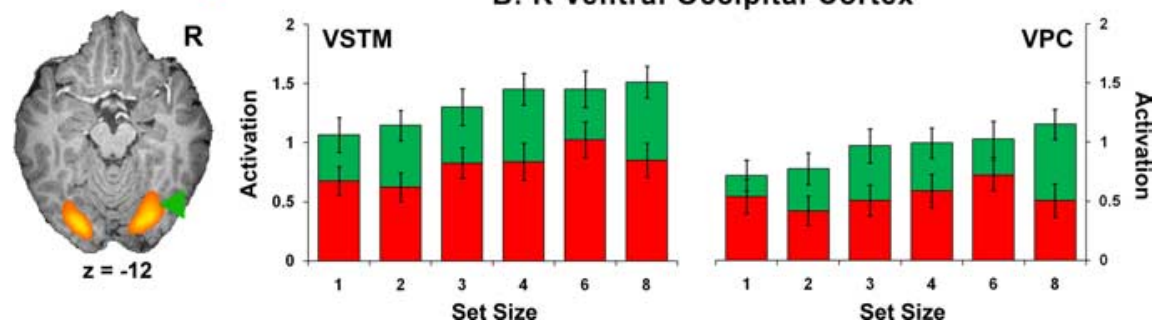

Figure 5. Mean activation ( \pm 1 SEM) in the IPS and ventral occipital cortex (V0) associated with the VSTM and VPC tasks as a function of state (RW, SD) and set size. There were significant effects of state and set size for the VSTM task, and significant effects of state for the VPC task in the IPS. In the V0, there were significant effects of state and set size for both tasks. Taking into consideration the entire cohort of 28 individuals, there was no main effect of drug or interaction effect for either task in both regions.

mine was associated with increased extrastriate activation but decreased prefrontal activation (Furey et al., 1997, 2000a,b).

\section{Discussion}

Cholinergic augmentation with donepezil reduced SD-induced decline in performance across both tasks and at all levels of memory or perceptual load in individuals who were vulnerable to performance decline when sleep deprived. It had a borderline detrimental effect in persons resistant to SD. Donepezil increased task-related activation in functionally relevant parieto-occipital areas in a manner that correlated with the modulation in performance. This provides converging evidence that donepezil can positively influence visual attention/processing in some sleepdeprived individuals and underscores the importance of considering interindividual differences when evaluating the outcome of manipulating neurotransmitter signaling.

\section{Multiple mechanisms through which cholinergic augmentation can affect sleep-deprived persons}

Increasing cholinergic transmission can benefit cognition in sleep-deprived individuals through four mechanisms: promoting wakefulness, increasing attention, increasing the signal-to-noise ratio of processing within visual sensory cortex, and promoting memory function in hippocampal circuits. The first three may be relevant in the current experiment.

We were initially motivated to evaluate the procognitive effects of donepezil in the context of SD on account of the role the cholinergic system plays in maintaining wakefulness (Everitt and Robbins, 1997; Steriade and McCarley, 2005) and sustained attention (Sarter et al., 2001). Sustained wakefulness is accompanied by elevation of adenosine levels, which in turn inhibits basal forebrain cholinergic output (Porkka-Heiskanen et al., 2002). However, based on subjective sleepiness scores, there was no evidence of a drug effect on perceived levels of wakefulness during $\mathrm{SD}$ in the current study.

Cholinergic afferents from the basal forebrain influence information processing in many cortical regions, including the parietal and extrastriate regions relevant to the present work (Voytko,
1996; Sarter and Bruno, 1997; Sarter et al., 2006). There is strong evidence linking visuospatial attention (Corbetta et al., 2000; Kastner and Ungerleider, 2000), sustained attention (Coull, 1998; Lawrence et al., 2003), and short-term visual storage (Todd and Marois, 2004) to the intraparietal sulcus. Application of the cholinergic antagonist scopolamine to this area impaired covert orienting (Davidson and Marrocco, 2000), whereas cholinergic augmentation with nicotine in healthy young adults (Lawrence et al., 2002; Thiel et al., 2005; Vossel et al., 2008) and physostigmine in patients with Alzheimer's disease (Bentley et al., 2008) improved performance and increased task-related activation. The higher parietal activation in persons who benefited from donepezil during SD in the present work suggests that modulation of attention might underlie the observed behavioral benefit.

Animal studies also indicate that acetylcholine is an important modulator of visual processing. Increasing cholinergic transmission may effect benefit directly by improving the signal-to-noise ratio of sensory processing (Sato et al., 1987; Murphy and Sillito, 1991) or indirectly through topdown effects (Sarter et al., 2001) mediated by prefrontal (Everitt and Robbins, 1997) and parietal cortices (Lawrence et al., 2002; Bentley et al., 2008). Human imaging studies are in agreement that the modulatory effect of cholinergic augmentation on blood flow is evident only when the visual task requires greater attention than the control task (Furey et al., 2000b; Lawrence et al., 2003; Bentley et al., 2004). Related to this, it was recently shown that, although SD is associated with profound hypoactivation of the extrastriate visual cortex when volunteers lapsed while performing a task engaging visual attention, the response of primary visual cortex to passive viewing of flickering check patterns was unaffected (Chee et al., 2008).

When working memory was evaluated using face stimuli, processing faces, as opposed to scrambled pictures, elicited higher MR signal in extrastriate cortex (Furey et al., 2000b). In another cohort, decreased extrastriate activation accompanied increased striate activation (Furey et al., 2008). Performance of a sequence detection task with a nicotine patch improved performance while increasing both parietal and visual extrastriate activation (Lawrence et al., 2003). Under the influence of physostigmine, extrastriate activation was higher during spatial working memory encoding (but not maintenance) and during a spatial attention task. Both these tasks were associated with reduced striate activation (Bentley et al., 2004). Whereas top-down effects arising from increased demands on attention might have been expected to raise parietal activation, in one of the experiments the reverse was observed.

A parsimonious explanation for these partially discordant findings concerning cholinergic effects appeared in a recent study that contrasted the effects of physostigmine on patients with early Alzheimer's disease and healthy age-matched controls (Bentley et al., 2008). Task-dependent activation in right parietal and posterior parahippocampal regions was lower in Alzheimer's disease patients, relative to controls, before physostigmine, but increased on its administration with accompanying behavioral benefit. Healthy controls did not benefit from augmentation and this was also reflected in 

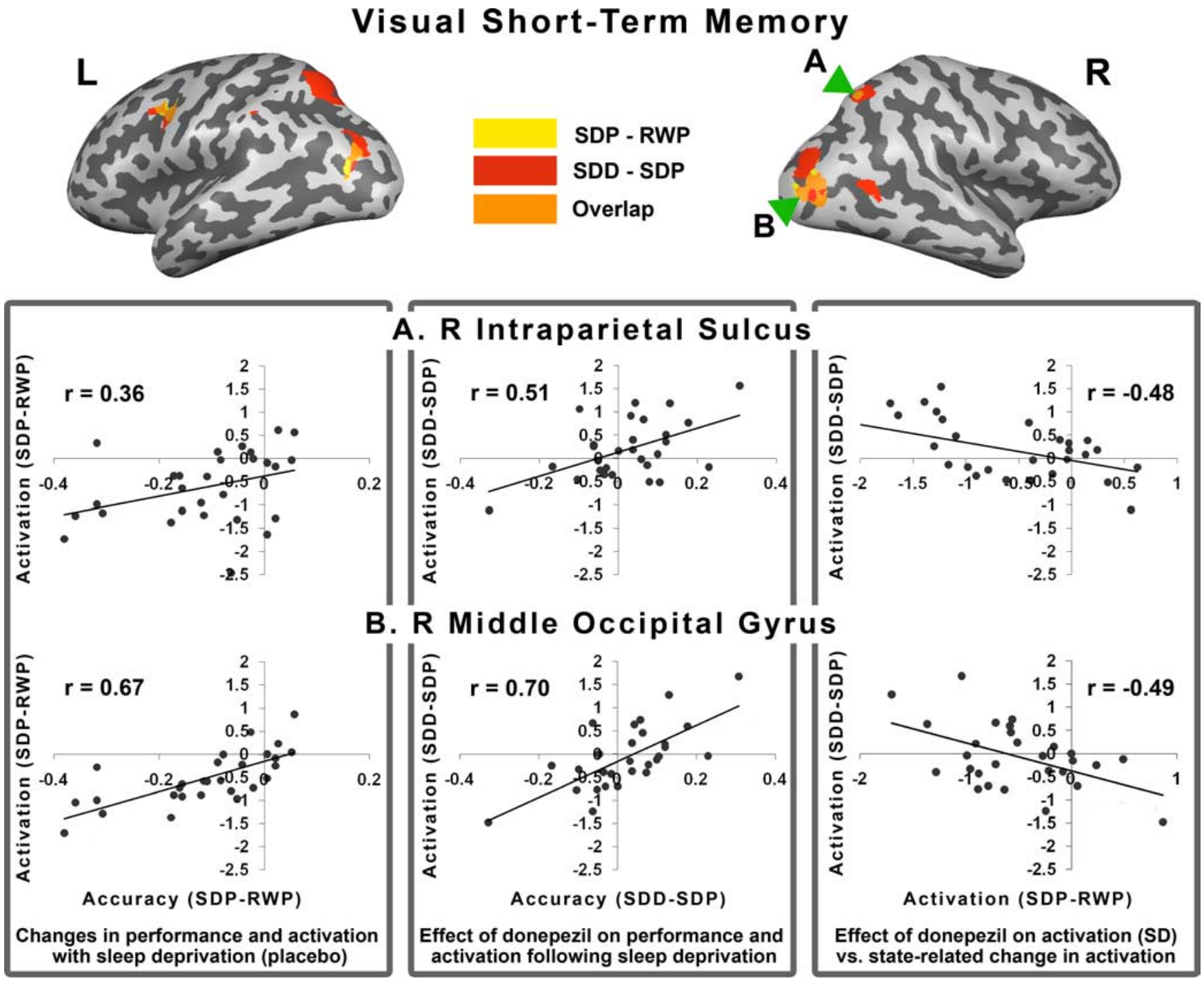

Figure 6. Top, The inflated brains show regions where activation tracked behavior decline after SD in the placebo condition (yellow). Regions in which activation tracked the effect of donepezil in the setting of SD are shown in red. The overlap of these regions appears in orange and data for the correlation plots that ensue were obtained from these regions. Bottom [left (L) to right (R)], The left panel shows the positive correlation between behavioral change elicited by SD in the placebo condition and the corresponding change in activation. The middle panel shows the correlation between how donepezil modulates performance and activation in the context ofSD. The right panel shows the correlation between donepezil-induced changes in activation in the context of SD with SD-induced changes in activation when volunteers were on placebo. All correlations were significant at $p<0.05$.

imaging. Because Alzheimer's disease is associated with deficient cholinergic transmission (Perry, 1986; Mesulam, 2004), it would appear that the existing level of cholinergic transmission influences how visual cortex activation is modulated (Bentley et al., 2008). Results from the present study buttress this proposition.

We hypothesized that a decline in cholinergic transmission might contribute to behavioral impairment in sleep-deprived persons. Persons more vulnerable to SD would then be expected to show a greater decline in cholinergic signaling when sleep deprived and, as a result, stand to benefit from donepezil. This was exactly what we found. Additionally, the behavioral benefit was positively correlated with parieto-occipital task-related activation, highlighting a compelling link between functional magnetic resonance imaging (fMRI) activation modulation and behavior. It follows that the behavioral and imaging consequences of cholinergic augmentation appear to depend on where an individual lies on the neurotransmitter-signaling continuum.

Conversely, one might expect performance decline and lower parieto-occipital activation in the low-vulnerable subjects after a normal night of sleep. However, although these individuals showed performance decline when sleep deprived on donepezil, they experienced no untoward effects of cholinergic augmentation after a normal night of sleep. As such, it is likely that, although the cholinergic system is important, it probably does not completely explain the current data.

Future work should examine the effects and interactions with other neurotransmitters known to modulate wakefulness and cognition. For example, it is known that cholinesterase inhibitors also affect noradrenergic and dopaminergic, in addition to cholinergic, transmission (Giacobini, 1996). Furthermore, donepezil and physostigmine (used in all fMRI studies on healthy young adults published to date) affect these transmitters to different degrees.

Interindividual differences in drug response and effects of sleep deprivation

The current results illustrate the feasibility of identifying behavioral and imaging correlates of interindividual variation in response to cognitive modulation in the setting of SD. There is good 


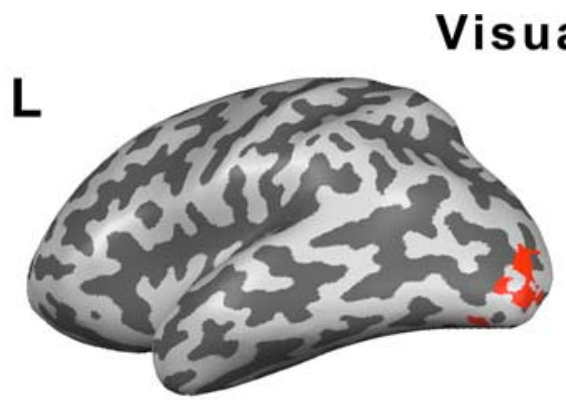

\section{Perceptual Control}

A. R Ventral Occipital Cortex
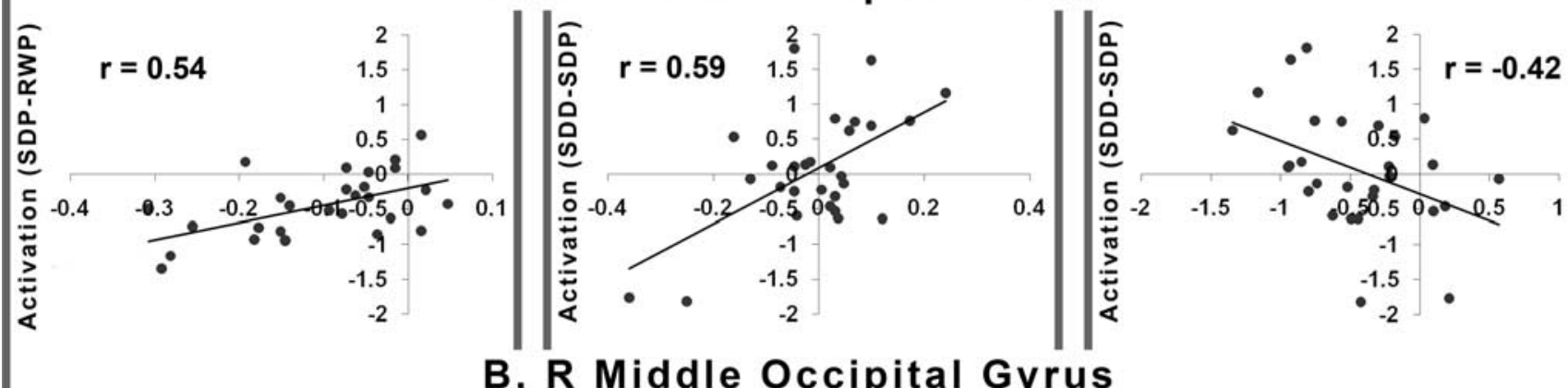

B. R Middle Occipital Gyrus

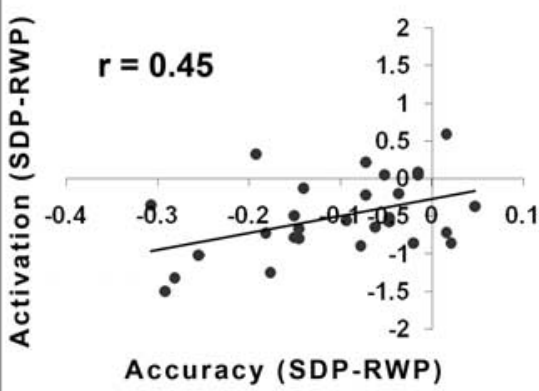

Changes in performance and activation with sleep deprivation (placebo)

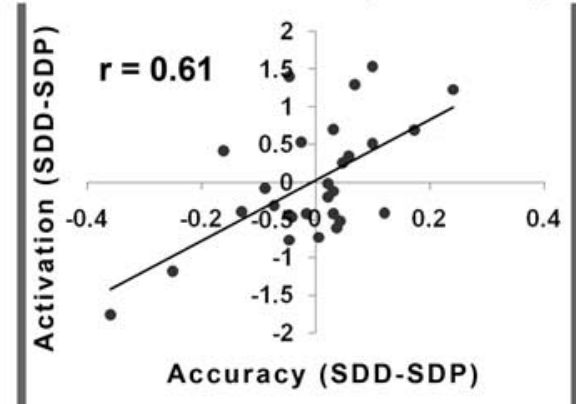

Effect of donepezil on performance and activation following sleep deprivation

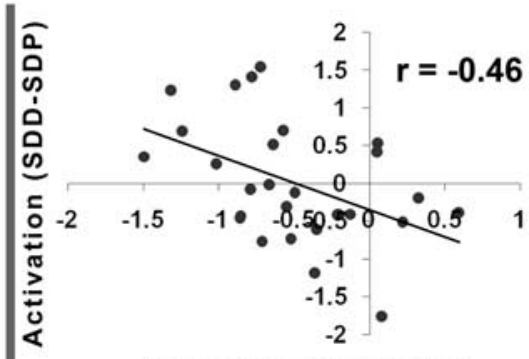

Activation (SDP-RWP)

Effect of donepezil on activation (SD) vs. state-related change in activation

Figure 7. Top, The inflated brains show regions in which activation tracked behavior decline after SD in the placebo condition (yellow). Regions in which activation tracked the effect of donepezil in the setting of SD are shown in red. The overlap of these regions appears in orange and data for the correlation plots that ensue were obtained from these regions. Bottom [left (L) to right (R)], The left panel shows the positive correlation between behavioral change elicited by SD in the placebo condition and the corresponding change in activation. The middle panel shows the correlation between how donepezil modulates performance and activation in the context of SD. The right panel shows the correlation between donepezil-induced changes in activation in the context of SD with SD-induced changes in activation when volunteers were on placebo. The right hemisphere was rotated slightly to expose the inferior surface of the occipital lobe. All correlations were significant at $p<0.05$.

behavioral evidence for the trait-like resistance to SD (Leproult et al., 2003; Van Dongen et al., 2004), although the genetic basis for such is only just emerging (Rétey et al., 2007; Viola et al., 2007). The characterization of neuroimaging correlates of vulnerability to SD is still preliminary (Mu et al., 2005; Chee et al., 2006) but appears encouragingly reproducible (Lim et al., 2007), an important feature when attempting to define an intermediate phenotype.

It is encouraging that interindividual differences in neural factors that adversely influence visual short-term memory and attention in the context of sleep deprivation appear to influence drug response in a manner that can be evaluated using functional brain imaging.

Specificity of drug and sleep deprivation effects on blood oxygen level-dependent signal

A potential confound in the present study is that, given the vasoactive properties of acetylcholine, cholinergic augmentation might be expected to increase blood flow in a nonspecific manner, for example, even at rest, as in the case of modafinil (Joo et al., 2008). However, previous studies have shown that cholinesterase inhibitors modulate blood flow in a task-dependent manner (Mentis et al., 2001; Bentley et al., 2004). In the current dataset, there are regions showing set size effects that do not show drug-induced modulation, for example, the pre-supplementary motor area.

An additional concern is that SD could by itself reduce taskrelated activation in a nonselective manner. Again, the findings of the current and previous functional imaging studies argue against this (Chee and Chuah, 2007), because there are areas showing task but not state effects, for example, in the primary visual cortex (Chee et al., 2008).

\section{Conclusions}

Donepezil can reduce the decline of visual short-term memory and visual attention in individuals relatively more vulnerable to 
the effects of sleep deprivation. However, the trend toward performance impairment in less vulnerable individuals should be noted. This behavioral benefit is accompanied by modulation of task-induced activation in the parieto-occipital cortex, suggesting that this behavioral benefit accrues from improvement in top-down control of visual attention, a direct effect on the extrastriate visual cortex or both these mechanisms.

\section{References}

Akerstedt T, Gillberg M (1990) Subjective and objective sleepiness in the active individual. Int J Neurosci 52:29-37.

Barger LK, Ayas NT, Cade BE, Cronin JW, Rosner B, Speizer FE, Czeisler CA (2006) Impact of extended-duration shifts on medical errors, adverse events, and attentional failures. PLoS Med 3:e487.

Beglinger LJ, Tangphao-Daniels O, Kareken DA, Zhang L, Mohs R, Siemers ER (2005) Neuropsychological test performance in healthy elderly volunteers before and after donepezil administration: a randomized, controlled study. J Clin Psychopharmacol 25:159-165.

Bentley P, Husain M, Dolan RJ (2004) Effects of cholinergic enhancement on visual stimulation, spatial attention, and spatial working memory. Neuron 41:969-982.

Bentley P, Driver J, Dolan RJ (2008) Cholinesterase inhibition modulates visual and attentional brain responses in Alzheimer's disease and health. Brain 131:409-424.

Bonnet MH, Balkin TJ, Dinges DF, Roehrs T, Rogers NL, Wesensten NJ (2005) The use of stimulants to modify performance during sleep loss: a review by the sleep deprivation and Stimulant Task Force of the American Academy of Sleep Medicine. Sleep 28:1163-1187.

Chee MW, Chuah LY (2008) Functional neuroimaging insights into how sleep and sleep deprivation affect memory and cognition. Curr Opin Neurol 21:417-423.

Chee MW, Chuah YM (2007) Functional neuroimaging and behavioral correlates of capacity decline in visual short-term memory following sleep deprivation. Proc Natl Acad Sci U S A 104:9487-9892.

Chee MW, Soon CS, Lee HL, Pallier C (2004) Left insula activation: a marker for language attainment in bilinguals. Proc Natl Acad Sci U S A 101:15265-15270.

Chee MW, Chuah LY, Venkatraman V, Chan WY, Philip P, Dinges DF (2006) Functional imaging of working memory following normal sleep and after 24 and 35 hours of sleep deprivation: correlations of frontoparietal activation with performance. Neuroimage 31:419-428.

Chee MW, Tan JC, Zheng H, Parimal S, Weissman DH, Zagorodnov V, Dinges DF (2008) Lapsing during sleep deprivation is associated with distributed changes in brain activation. J Neurosci 28:5519-5528.

Corbetta M, Kincade JM, Ollinger JM, McAvoy MP, Shulman GL (2000) Voluntary orienting is dissociated from target detection in human posterior parietal cortex. Nat Neurosci 3:292-297.

Coull JT (1998) Neural correlates of attention and arousal: insights from electrophysiology, functional neuroimaging and psychopharmacology. Prog Neurobiol 55:343-361.

Cowan N (2001) The magical number 4 in short-term memory: a reconsideration of mental storage capacity. Behav Brain Sci 24:87-114; discussion $114-185$.

Davidson MC, Marrocco RT (2000) Local infusion of scopolamine into intraparietal cortex slows covert orienting in rhesus monkeys. J Neurophysiol 83:1536-1549.

Dinges DF (1995) An overview of sleepiness and accidents. J Sleep Res $4: 4-14$.

Dinges DF, Pack F, Williams K, Gillen KA, Powell JW, Ott GE, Aptowicz C, Pack AI (1997) Cumulative sleepiness, mood disturbance, and psychomotor vigilance performance decrements during a week of sleep restricted to 4-5 hours per night. Sleep 20:267-277.

Doran SM, Van Dongen HP, Dinges DF (2001) Sustained attention performance during sleep deprivation: evidence of state instability. Arch Ital Biol 139:253-267.

Durmer JS, Dinges DF (2005) Neurocognitive consequences of sleep deprivation. Semin Neurol 25:117-129.

Everitt BJ, Robbins TW (1997) Central cholinergic systems and cognition. Annu Rev Psychol 48:649-684.

Furey ML, Pietrini P, Haxby JV, Alexander GE, Lee HC, VanMeter J, Grady CL, Shetty U, Rapoport SI, Schapiro MB, Freo U (1997) Cholinergic stimulation alters performance and task-specific regional cerebral blood flow during working memory. Proc Natl Acad Sci U S A 94:6512-6516.

Furey ML, Pietrini P, Alexander GE, Schapiro MB, Horwitz B (2000a) Cholinergic enhancement improves performance on working memory by modulating the functional activity in distinct brain regions: a positron emission tomography regional cerebral blood flow study in healthy humans. Brain Res Bull 51:213-218.

Furey ML, Pietrini P, Haxby JV (2000b) Cholinergic enhancement and increased selectivity of perceptual processing during working memory. Science 290:2315-2319.

Furey ML, Ricciardi E, Schapiro MB, Rapoport SI, Pietrini P (2008) Cholinergic enhancement eliminates modulation of neural activity by task difficulty in the prefrontal cortex during working memory. J Cogn Neurosci 20:1342-1353.

Giacobini E (1996) Cholinesterase inhibitors do more than inhibit cholinesterase. In: Alzheimer disease: from molecular biology to therapy (Becker R, Giacobini E, eds), pp 187-204. Boston: Birkhauser.

Goebel R, Esposito F, Formisano E (2006) Analysis of functional image analysis contest (FIAC) data with brainvoyager QX: from single-subject to cortically aligned group general linear model analysis and self-organizing group independent component analysis. Hum Brain Mapp 27:392-401.

Gray JR, Chabris CF, Braver TS (2003) Neural mechanisms of general fluid intelligence. Nat Neurosci 6:316-322.

Habeck C, Rakitin BC, Moeller J, Scarmeas N, Zarahn E, Brown T, Stern Y (2004) An event-related fMRI study of the neurobehavioral impact of sleep deprivation on performance of a delayed-match-to-sample task. Brain Res Cogn Brain Res 18:306-321.

Honey G, Bullmore E (2004) Human pharmacological MRI. Trends Pharmacol Sci 25:366-374.

Horne JA, Ostberg O (1976) A self-assessment questionnaire to determine morningness-eveningness in human circadian rhythms. Int J Chronobiol 4:97-110.

Horne JA, Reyner LA (1995) Sleep related vehicle accidents. BMJ 310:565-567.

Joo EY, Tae WS, Jung KY, Hong SB (2008) Cerebral blood flow changes in man by wake-promoting drug, modafinil: a randomized double blind study. J Sleep Res 17:82-88.

Kastner S, Ungerleider LG (2000) Mechanisms of visual attention in the human cortex. Annu Rev Neurosci 23:315-341.

Lawrence NS, Ross TJ, Stein EA (2002) Cognitive mechanisms of nicotine on visual attention. Neuron 36:539-548.

Lawrence NS, Ross TJ, Hoffmann R, Garavan H, Stein EA (2003) Multiple neuronal networks mediate sustained attention. J Cogn Neurosci 15:1028-1038.

Leproult R, Colecchia EF, Berardi AM, Stickgold R, Kosslyn SM, Van Cauter E (2003) Individual differences in subjective and objective alertness during sleep deprivation are stable and unrelated. Am J Physiol Regul Integr Comp Physiol 284:R280-R290.

Lim J, Dinges DF (2008) Sleep deprivation and vigilant attention. Ann N Y Acad Sci 1129:305-322.

Lim J, Choo WC, Chee MW (2007) Reproducibility of changes in behavior and fMRI activation associated with sleep deprivation in a working memory task. Sleep 30:61-70.

Linden DE, Bittner RA, Muckli L, Waltz JA, Kriegeskorte N, Goebel R, Singer W, Munk MH (2003) Cortical capacity constraints for visual working memory: dissociation of fMRI load effects in a fronto-parietal network. Neuroimage 20:1518-1530.

Luck SJ, Vogel EK (1997) The capacity of visual working memory for features and conjunctions. Nature 390:279-281.

Majerus S, Bastin C, Poncelet M, Van der Linden M, Salmon E, Collette F, Maquet P (2007) Short-term memory and the left intraparietal sulcus: focus of attention? Further evidence from a face short-term memory paradigm. Neuroimage 35:353-367.

Mentis MJ, Sunderland T, Lai J, Connolly C, Krasuski J, Levine B, Friz J, Sobti S, Schapiro M, Rapoport SI (2001) Muscarinic versus nicotinic modulation of a visual task. A PET study using drug probes. Neuropsychopharmacology 25:555-564.

Mesulam M (2004) The cholinergic lesion of Alzheimer's disease: pivotal factor or side show? Learn Mem 11:43-49.

Mitchell DJ, Cusack R (2007) Flexible, capacity-limited activity of posterior parietal cortex in perceptual as well as visual short-term memory tasks. Cereb Cortex 18:1788-1798. 
Mitler MM, Carskadon MA, Czeisler CA, Dement WC, Dinges DF, Graeber RC (1988) Catastrophes, sleep, and public policy: consensus report. Sleep 11:100-109.

Mu Q, Mishory A, Johnson KA, Nahas Z, Kozel FA, Yamanaka K, Bohning DE, George MS (2005) Decreased brain activation during a working memory task at rested baseline is associated with vulnerability to sleep deprivation. Sleep 28:433-446.

Murphy PC, Sillito AM (1991) Cholinergic enhancement of direction selectivity in the visual cortex of the cat. Neuroscience 40:13-20.

Perry EK (1986) The cholinergic hypothesis-ten years on. Br Med Bull 42:63-69.

Philip P, Akerstedt T (2006) Transport and industrial safety, how are they affected by sleepiness and sleep restriction? Sleep Med Rev 10:347-356.

Phillips JM, McAlonan K, Robb WG, Brown VJ (2000) Cholinergic neurotransmission influences covert orientation of visuospatial attention in the rat. Psychopharmacology (Berl) 150:112-116.

Porkka-Heiskanen T, Alanko L, Kalinchuk A, Stenberg D (2002) Adenosine and sleep. Sleep Med Rev 6:321-332.

Rétey JV, Adam M, Khatami R, Luhmann UF, Jung HH, Berger W, Landolt $\mathrm{HP}$ (2007) A genetic variation in the adenosine A2A receptor gene (ADORA2A) contributes to individual sensitivity to caffeine effects on sleep. Clin Pharmacol Ther 81:692-698.

Rypma B, Berger JS, Prabhakaran V, Bly BM, Kimberg DY, Biswal BB, D'Esposito M (2006) Neural correlates of cognitive efficiency. Neuroimage 33:969-979.

Sarter M, Bruno JP (1997) Cognitive functions of cortical acetylcholine: toward a unifying hypothesis. Brain Res Brain Res Rev 23:28-46.

Sarter M, Givens B, Bruno JP (2001) The cognitive neuroscience of sus- tained attention: where top-down meets bottom-up. Brain Res Brain Res Rev 35:146-160.

Sarter M, Gehring WJ, Kozak R (2006) More attention must be paid: the neurobiology of attentional effort. Brain Res Rev 51:145-160.

Sato H, Hata Y, Masui H, Tsumoto T (1987) A functional role of cholinergic innervation to neurons in the cat visual cortex. J Neurophysiol 58:765-780.

Steriade M, McCarley RW (2005) Changing concepts of mechanisms of waking and sleep states. In: Brain control of wakefulness and sleep, pp 1-33. New York: Kluwer Academic.

Thiel CM, Zilles K, Fink GR (2005) Nicotine modulates reorienting of visuospatial attention and neural activity in human parietal cortex. Neuropsychopharmacology 30:810-820.

Thomas RJ, Kwong K (2006) Modafinil activates cortical and subcortical sites in the sleep-deprived state. Sleep 29:1471-1481.

Todd JJ, Marois R (2004) Capacity limit of visual short-term memory in human posterior parietal cortex. Nature 428:751-754.

Van Dongen HP, Baynard MD, Maislin G, Dinges DF (2004) Systematic interindividual differences in neurobehavioral impairment from sleep loss: evidence of trait-like differential vulnerability. Sleep 27:423-433.

Viola AU, Archer SN, James LM, Groeger JA, Lo JC, Skene DJ, von Schantz M, Dijk DJ (2007) PER3 polymorphism predicts sleep structure and waking performance. Curr Biol 17:613-618.

Vossel S, Thiel CM, Fink GR (2008) Behavioral and neural effects of nicotine on visuospatial attentional reorienting in non-smoking subjects. Neuropsychopharmacology 33:731-738.

Voytko ML (1996) Cognitive functions of the basal forebrain cholinergic system in monkeys: memory or attention? Behav Brain Res 75:13-25. 